\title{
Effects of Solar Eclipse on Audio Frequency Atmospherics
}

By Everetr T. Burton and Edward M. Boardman, Bell Telephone Laboratories, New York City

STUDIES of low-frequency atmospherics have $S$ shown marked diurnal variations, the nighttime intensity considerably exceeding that of the day. ${ }^{1}$ These variations may be attributed to radiation from the sun through its effect on ionisation in the reflecting and absorbing layers of the upper atmosphere. Although it is believed that the major portion of this ionisation is due to sunlight, an appreciable effect may be due to a eorpuscular radiation from the sun. A solar eclipse affords an opportunity for comparing light and corpuscular effects, since a difference in velocity of these types of radiation should result in a time separation of the two eclipses at the earth's surface. Prof. S. Chapman ${ }^{2}$ has shown that, with an assumed corpuscular velocity of 1,000 miles a second, the corpuscular eclipse precedes the visible one by about two hours. The motion of the moon through a corpuscular radiation results in a trailing shadow. In overtaking the visible shadow of the moon, the earth arrives first at the location of the corpuscular shadow.

Observations of atmospherics during the progress of a solar eclipse should show at least an approximation to night-time conditions, because of the accompanying reduction of radiation intensity. The effects of the two types of radiation on the ionisation in the atmosphere should be sufficiently separated in time to render their effects

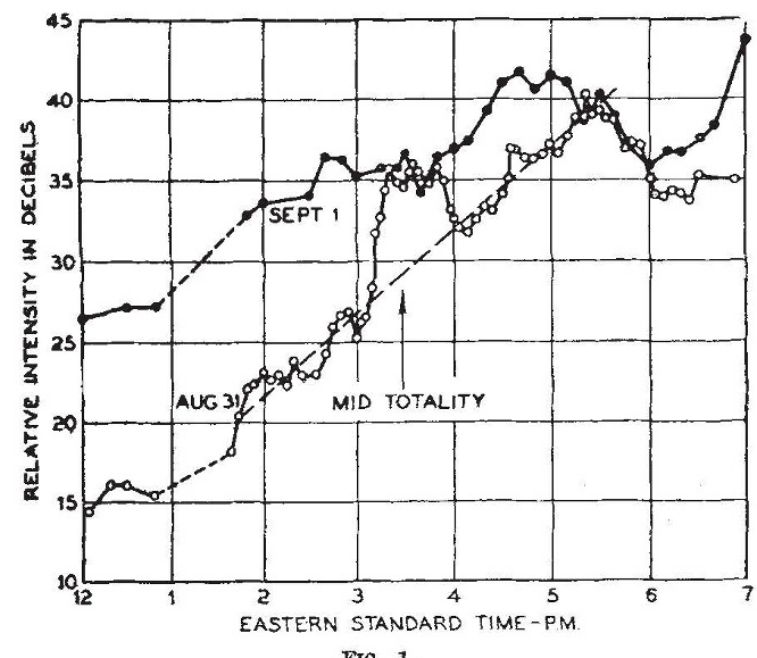

FIG. 1.

distinct. To obtain experimental data, apparatus was set up at Conway, New Hampshire, which was in the path of totality of the solar eclipse of August 31, 1932. A vacuum tube amplifier was connected to a vertical loop antenna which consisted of two sections placed at right angles to reduce directional effects. Between the loop and the amplifier were placed networks so designed that all frequencies above the range of interest were eliminated, thus insuring direct reception of audio frequencies without a detection process occurring anywhere in the apparatus. The amplifier output was connected to a thermocouple for amplitude measurement and to a telephone for aural observations. The amplifier gain was essentially uniform from 20 to 3,800 cycles per second.

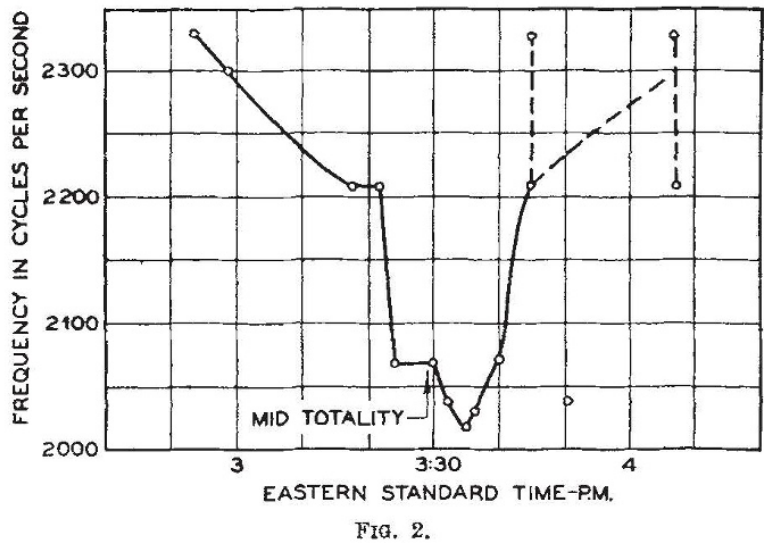

The telephones were of the dynamic type with regular response throughout the voice range. These permitted more accurate observations on static qualities than have been possible with the telephone receivers used heretofore. A calibrated tone circuit, loosely coupled to the amplifier system, was provided for use in determining frequencies of musical atmospherics.

Tests at Conway extended from the evening of August 30 until the morning of September 2. Frequent determinations of static amplitude and of static tonal qualities were made. The atmospheric intensity during all of this time was unusually high because of the presence of musical atmospherics of the previously reported type known as 'swish'. While the 'swish' obviously was not related to the eelipse, its presence was unfortunate because of the introduction of irregular variations of intensity. The static intensities for the afternoons of August 31 and September 1 are shown in Fig. 1. While the two curves follow the same general contour, that of the former date shows an abrupt rise shortly before the time of eclipse totality and an abrupt fall after totality. The duration of this period of high intensity is 55 minutes, its mid-time occurring 5 minutes after mid-totality.

Certain types of so-called 'musical atmospherics' normally occur only during twilight or night. One of these, known as the 'tweek', is a short damped oscillation which appears to result from multiple reflections of a static impulse between the earth's surface and a reflecting layer located in the upper atmosphere. 'Tweeks' have never been observed during full daylight, their existence evidently being prevented by absorption in the low-altitude 
ionisation maintained by sunlight. A phenomenon closely related to the 'tweek' is a weak resonance, observed during evening and morning twilight periods. The resonance has been found to appear near sunset with a frequency of approximately 2,300 cycles per second, reducing gradually to 1,800 cycles per second in approximately two and a half hours, after which it appears to sub. merge in the night-time static. During the morning twilight period the resonance reappears, rising through the same frequency range and persisting for approximately half an hour. The 'tweeks' suffer similar frequency variations during the twilight periods.

A watch was kept for tonal qualities in the static during the entire day of the eclipse. Shortly after noon, occasional very highly damped oscillations were observed, resembling somewhat the nighttime 'tweeks'. Seventeen of these were observed between the hours of 12.35 and 3.50 P.M., a good share of them occurring just previous to one o'clock. While our data are meagre, there is evidence of a maximum in this effect occurring somewhere between two and three quarters and one and a half hours before the time of visible totality. This uncertainty results from the fact that no observations of tone were made between the hours of one and two in the afternoon. As totality approached these highly damped tones were seldom observed, but a weak resonance similar to that of an evening twilight period appeared. The frequency was observed to reduce gradually, reaching its minimum approximately seven minutes after totality. Thereafter it increased rapidly, and disappeared after a short period of rather indefinite frequency. The observations of the resonance tone are shown in Fig. 2.

Our observations of audio-frequency atmospheric intensity indicate an approach to night-time intensity near the period of totality. Data on resonance tone indicate an approximation to evening conditions before and during totality, followed by a condition similar to that of morning. Both the intensity and tonal data show lags of a few minutes in respect to the time of totality. These observations point to sunlight effects on the ionisation of the atmosphere. The occasional occurrence of the highly damped tones long before the time of visible totality may be taken as evidence of the existence of corpuscular radiation, although the data indicate a minor effect. The difference in tonal quality between the early highly damped oscillations and the resonance observed near the time of visible totality indicates the occurrence of some marked change in the atmospheric conducting or reflecting properties, due possibly to difference in penetrating properties of the two types of radiation.

${ }^{1}$ E. T. Burton, "Submarine Cable Interference", NatuRE, 126, 55, July 12, 1930.

'S. Chapman, "Influence of Solar Eclipse Upon Upper Atmospheric Ionization", Mon. Not. Roy. Ast. Soc., 92, 413; 1932.

\section{Infestation of Stored Products by Insects}

\section{By Prof. J. W. Munro, Imperial College of Science and Technology}

$A^{\text {LTHOUGH the losses caused to growing }}$ $\mathrm{A}_{\text {crops by insects have long been recognised, }}$ the losses caused to crops after harvesting and during storage and transport have been strangely neglected both by the biologist and the industrialist. These losses are none the less of grave importance and take in the main three forms: absolute loss of goods resulting from consumption by the insect, indirect loss resulting from the lower price paid for soiled or infested produce and indirect loss resulting from loss of prestige by the merchant or manufacturer who inadvertently places infested produce on the market. This last form of loss is almost wholly incalculable, but that it is high is evident from the almost extravagant care which our food industries take to prevent any knowledge of infestation from reaching the general public.

In 1926, the Empire Marketing Board appointed a committee-Committee on Stored Products Infestation-to consider this problem and on its recommendation made a grant to the Imperial College, in 1927, for the establishment of a special laboratory for the study of the scientific problems underlying infestation of stored products by insects and fungi.* This laboratory is stationed

* The term 'stored products' as used here excludes timber, which is dealt with by the Forest Products Research Laboratory of the Department of Scientific and Industrial Research. at Slough, near London. Its work is divisible into three main sections: survey and intelligence work at the London docks; research and experimental work in the laboratory; and commercial scale experimental work on sterilisation of infested produce and warehouses in London and other ports.

The survey or intelligence work is concerned with inspection of such produce as cocoa ${ }^{1}$, dried fruits $^{2}$ and tobacco ${ }^{3}$ on its arrival in London, with a study of the special conditions-economic and climatic - prevailing in the dock warehouses and their relationship to infestation, and with the examination of special 'experimental consignments' of produce shipped from oversea for the purpose of testing methods of preparation, packing and transport and their relationship to insect infestation. With this inspection work there is also regular correspondence and exchange of programmes with departments of agriculture and other institutions in the Dominions and Colonies on problems arising in the work.

A survey of the insects associated with cocoa, dried fruits, spices and tobacco stored in London warehouses showed that upwards of a hundred and forty species of insect are represented, but of these only a few are of primary importance'. ${ }^{4}$ These are the phycitid moths of the genera Ephestia 